\title{
Weibel Instability in a Bi-Maxwellian Laser Fusion Plasma
}

\author{
Abdelaziz SID, Abdennasser GHEZAL ${ }^{1)}$, Azzeddine SOUDANI ${ }^{2)}$ and Mohamed BEKHOUCHE \\ Laboratoire de Physique des Rayonnements et leurs interactions avec la Matière (PRIMALAB), \\ Département de Physique, Faculté des Sciences, Université de Batna, Batna, 05000 DZ, Algeria \\ ${ }^{1)}$ Commissariat à l'Energie Atomique, Centre de Recherche Nucléaire de Draria, \\ Division de Sûreté Nucléaire et Radioprotection, Draria, Alger, Algeria \\ ${ }^{2)}$ Laboratoire de Physique Energétique Appliquée, Département de Physique, \\ Faculté des Sciences, Université de Batna, Batna, 05000 DZ, Algeria
}

(Received 13 November 2009 / Accepted 14 January 2010)

In this paper, the Weibel instability, driven by the plasma temperature anisotropy, in the corona of high intense laser fusion plasma is studied. The unperturbed electronic distribution function, $f$, of the anisotropic corona is supposed to be a bi-Maxwellian. That $T_{\|}=T_{\perp} \pm W_{\mathrm{O}}$, where $W_{\mathrm{O}}=\frac{1}{4} m_{\mathrm{e}} v_{\mathrm{O}}^{2}$ is the averaged electron quiver energy in the laser electric field. The first and the second anisotropies of $f$ projected on the Legendre polynomials are calculated as a function of the scaling parameter, $\frac{W_{O}}{T_{\perp}}$. The Weibel instability parameters are explicitly calculated as a function of the scaling parameter. For typical parameters of the laser pulse and the fusion plasma, it has been shown that very unstable Weibel modes, $\gamma \gtrsim 10^{11} \mathrm{~s}^{-1}$, can be excited in the corona.

(C) 2010 The Japan Society of Plasma Science and Nuclear Fusion Research

Keywords: inertial fusion, laser created plasma, electron oscillation, Weibel instability, scaling law

DOI: $10.1585 /$ pfr.5.007

\section{Introduction}

Erich Weibel[1] was the first who predicted the spontaneously growing of the transverse quasi-static electromagnetic waves which appear in plasma, due to an anisotropic velocity distribution of electrons. The maximum increment of this instability for the wave frequency $\omega$ is $\gamma=\operatorname{Im}(\omega)=\frac{u}{c} \omega_{\mathrm{p}}$, where $\omega_{\mathrm{p}}$ is the plasma frequency and $u$ is the average velocity of electrons in the anisotropic direction. This simple result is valid under the condition of a strong anisotropy of the velocity distribution and small wavelengths electromagnetic waves $\left(k c \gg \omega_{\mathrm{p}}\right.$, where $k$ is the wave number). This velocity anisotropy can be interpreted, in macroscopic level, as anisotropy in the plasma temperature.

The Weibel instability is intensively studied for both the astrophysical plasma [2] and the fusion plasma [3-7]. In the laser fusion plasma, several mechanisms contribute to the excitation of the Weibel instability; namely the temperature gradient, the plasma expansion, the inverse bremsstrahlung... etc.

In the inertial confinement fusion (ICF) targets, produced by an intense laser pulse, the incident laser wave [8-10] produces anisotropy in the formed plasma temperature. This is due to the fact that the plasma is preferentially heated in the direction of the laser wave electric field. It has been shown that this anisotropic distribution provokes unstable Weibel electromagnetic modes [3-7]. If this instability is excited, strong (giga gauss) magnetic fields can be generated from the magnetic fields germ due to the elec-

author'se-mail: a_sid@univ-batna.dz,sid_abdelaziz@hotmail.com tron thermal motion.

In the laser fusion experiments, the Weibel instability inhibits the heat flux and influences negatively on the implosion characteristics of the target. This may have a possibility to give rise to energy loss.

This paper deals with the theoretical study of the Weibel instability excited in the laser fusion plasma corona. In our model the unstable Weibel modes are excited by the direct effect of the laser electric field on the coronal plasma. That the corona is characterized by a plasma frequency, $\omega_{\mathrm{p}}$, less than the laser wave frequency, $\omega_{\mathrm{L}}: \omega_{\mathrm{p}}<\omega_{\mathrm{L}}$.

The present work is organized as follows: in Sec. 2, we present the electronic distribution function which is supposed to be a local bi-Maxwellian. In Sec. 3, we present a theoretical analysis of the Weibel modes. The Sec. 4 is devoted to the scaling laws for the instability parameters. Finally, a conclusion for the obtained results is given.

\section{Distribution Function}

In our model, the electronic distribution function, $f$, is assumed to be a local bi-Maxwellian, where the anisotropy in electron temperature is due to the direct effect of the laser electric field on the corona electrons. This is justified by the fact that the plasma is preferentially heated in the direction of the laser electric field, so:

$f=\left(\frac{m_{\mathrm{e}}}{2 \pi}\right)^{3 / 2} \frac{n_{\mathrm{e}}}{T_{\perp} T_{\|}^{1 / 2}} \exp \left(-\frac{1}{2} \frac{m_{\mathrm{e}} v_{\perp}^{2}}{T_{\perp}}\right) \exp \left(-\frac{1}{2} \frac{m_{\mathrm{e}} v_{\|}^{2}}{T_{\|}}\right)$,

where $e, m_{\mathrm{e}}, T_{\|}$and $T_{\perp}$ are respectively the elementary 
electric charge, the electron mass, the electron density, the electron temperature in the anisotropy direction and the electron temperature in the perpendicular plane.

In the case of the linear polarized laser pulse, with the laser wave electric field oriented in the parallel direction, the temperature anisotropy is given by: $\Delta T=T_{\|}-T_{\perp}=$ $W_{\mathrm{O}}$, where $W_{\mathrm{O}}$ is the average, on the laser cycle, of the oscillating energy communicated to the electron by the laser wave. In this case, the parallel Weibel mode, $k=k_{\|}$, is excited. However, in the case of circularly polarized laser wave, with the laser wave electric field oscillating in the perpendicular plane, the temperature anisotropy is given by: $\Delta T=T_{\perp}-T_{\|}=W_{\mathrm{O}}$. In this case, the perpendicular Weibel mode, $k=k_{\perp}$, is excited.

The average electron quiver energy, $W_{\mathrm{O}}$, in both polarization cases, represents the anisotropy in the electron temperature. It is calculated using the perturbed fluid electron motion equation by considering the collisions [11-13], so:

$$
\frac{\partial v_{\mathrm{o}}}{\partial t}=-\frac{e}{m_{\mathrm{e}}} E-v_{\mathrm{c}} v_{\mathrm{o}}
$$

where $v_{\mathrm{o}}, E$ and $v_{\mathrm{c}}$ are respectively the electron quiver velocity, the laser electric field magnitude and the collision frequency.

Not that in the above equation, the force due to pressure gradient is dropped because it vanishes at the first order in the case of plasma perturbed by an electromagnetic wave. This is justified by the fact that the electromagnetic perturbation has no effect on the electron density. Also, the temporal variation of the perturbed quantities follows the temporal variation of the laser electric field which is assumed as a normal mode, $E \sim \exp \left(i \omega_{\mathrm{L}} t\right)$. Therefore, the average electron quiver energy on a laser wave cycle, $W_{\mathrm{O}}=\frac{1}{4} m_{\mathrm{e}}\left|v_{\mathrm{o}}\right|^{2}$, can be presented as:

$$
W_{\mathrm{O}}=\frac{e^{2}}{2 \varepsilon_{0} c m_{\mathrm{e}}} \frac{I}{\omega_{\mathrm{L}}^{2}}\left[1-\left(\frac{v_{\mathrm{c}}}{\omega_{L}}\right)^{2}\right],
$$

where $\varepsilon_{0}, c$ and $I=\frac{1}{2} c \varepsilon_{0}|E|^{2}$ are the vacuum electric permittivity, the speed of light in vacuum and the local laser pulse intensity respectively.

Equation (2) shows that $W_{\mathrm{O}}$ decreases as the collision frequency increases. This is interpreted by the fact that the electron temperature undergoes an isotropisation due to collisions. Then, we expect that collisions decrease the Weibel instability growth rate (Sec. 3).

The collision frequency, $v_{\mathrm{c}} \sim n_{\mathrm{e}} /(T)^{3 / 2}$, corresponds to the electron-ion collision frequency according to the Lorentz approximation, that is justified in the laser fusion plasma corona, where $n_{\mathrm{e}}$ is the electron density and $T$ is the electron temperature:

$$
T=\int_{0}^{\infty}\left(\frac{m_{\mathrm{e}}}{2} v^{2}\right) f \mathrm{~d}^{3} \vec{v} \mid \int_{0}^{\infty} f \mathrm{~d}^{3} \vec{v}
$$

It is necessary to calculate the three first components of the unperturbed electron distribution function, truncated on the Legendre polynomials that the Weibel instability parameters depend on these functions (Sec.3). We develop the bi-Maxwellian electronic distribution function (Eq. (1)) on the Legendre polynomials [14], $P_{l}\left(\mu=\frac{v_{\|}}{v}\right)$ : $f=\sum_{0}^{\infty} P_{l}(\mu) f_{l}(v)$. Hence, the isotropic distribution function, $f_{0}$, the first anisotropic function, $f_{1}$, and the second anisotropic function, $f_{2}$, are calculated as:

$$
\begin{aligned}
f_{0}= & \left(\frac{m_{\mathrm{e}}}{2 \pi T_{\perp}}\right)^{3 / 2} n_{\mathrm{e}} \exp (-y) \\
& \times\left\{\left(1+\widetilde{W}_{\mathrm{O}}\right)^{-1 / 2}+\frac{y}{3} \widetilde{W}_{\mathrm{O}}\left(1+\widetilde{W}_{\mathrm{O}}\right)^{-3 / 2}\right\}, \\
f_{1}= & 0, \\
f_{2}= & \left(\frac{m_{\mathrm{e}}}{2 \pi T_{\perp}}\right)^{3 / 2} n_{\mathrm{e}} \exp (-y)\left\{\frac{2 y}{3} \widetilde{W}_{\mathrm{O}}\left(1+\widetilde{W}_{\mathrm{O}}\right)^{-1 / 2}\right\},
\end{aligned}
$$

where $y=\frac{m_{\mathrm{e}} \mathrm{v}^{2}}{2 T_{\perp}}$ and $\widetilde{W}_{\mathrm{O}}=\frac{W_{\mathrm{O}}}{T_{\perp}}$. The scaling parameter $\widetilde{W}_{\mathrm{O}}$ measures the direct effect of the laser pulse on the electrons. In the same time it measures the anisotropy in temperature which is the source of the Weibel instability.

We have presented, on the Fig. 1, the plot of $f_{0}(y)$ and $f_{2}(y)$ for several values of $\widetilde{W}_{\mathrm{O}}$.

We point out from these that the higher components of unperturbed distribution became important as the scaling parameter increases.

\section{Weibel Instability Analysis}

The Weibel instability is a micro-instability which needs a kinetic analysis. However, the analytical establishment of a dispersion relation, which takes into account the collisions, from the perturbed Fokker-Planck equation is difficult. This is due principally to the nonlinearity of the collisions term.

Several approaches to the Weibel dispersion relation in the laser created plasma are reported in the literature. For example, in Ref. [4], Wallace and Epperlein have established a dispersion relation from the perturbed FokkerPlanck equation in the collisional limit, where the wavelength of the excited Weibel mode is greater than the main free path of electron. However, in the Ref. [5] Ramani and Laval have established a dispersion relation valid in the collisionless limit. We found that the growth rate value calculated by using the dispersion relation of Ref. [4] is reduced compared to that calculated by using the dispersion relation of the Ref. [5]. This is in agreement with the prediction of the Ref. [15].

In the Ref. [16], a dispersion relation, in the semicollisional regime, is established. This dispersion relation is derived in the Lorentz gas and in the local approximations. It is valid in the whole collisionality regime. The group velocity and the growth rate of the $k$ Weibel mode are given by:

$$
v_{\mathrm{g}}(k)=\left[\frac{\sqrt{3}}{2} v_{\mathrm{t}} \int_{0}^{\infty} y^{5 / 2} F(y, k \lambda) f_{1} \mathrm{~d} y\right] / D
$$


(a)

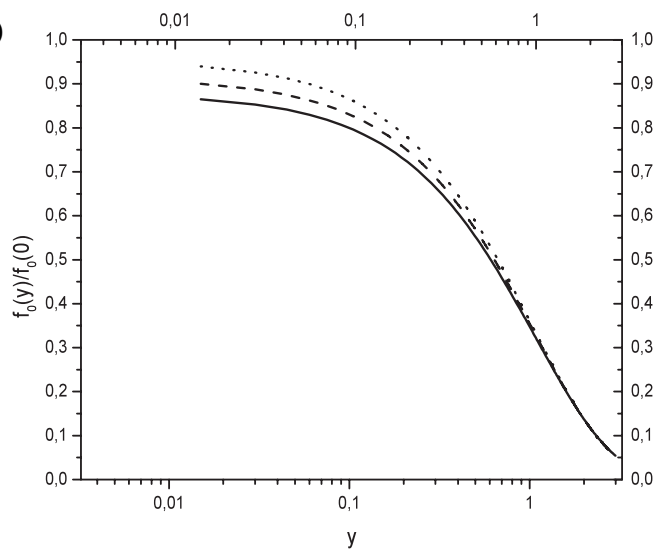

(b)

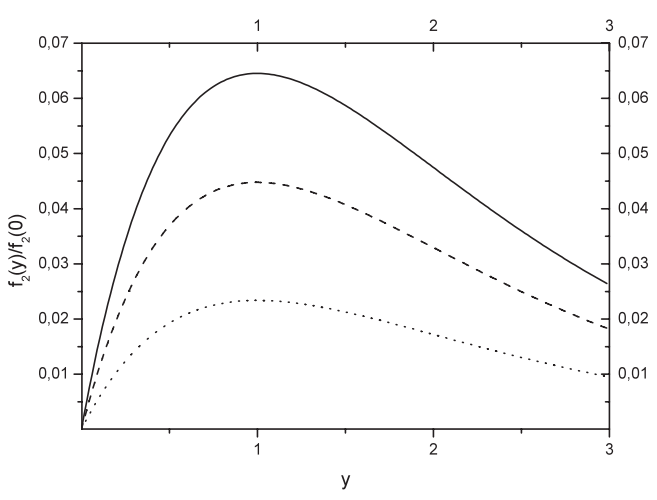

Fig. 1 (a) Isotropic distribution function, $f_{0}$, as function of $y$ for several values of the scaling parameter, $\widetilde{W}_{\mathrm{O}}$. The dotted curve corresponds to $\widetilde{W}_{\mathrm{O}}=0.1$, the dashed curve corresponds to $\widetilde{W}_{\mathrm{O}}=0.2$ and the solid one corresponds to $\widetilde{W}_{\mathrm{O}}=0.3$.

(b) Second anisotropic distribution function, $f_{2}$, as function of $y$ for several values of the scaling parameter, $\widetilde{W}_{\mathrm{O}}$. The dotted curve corresponds to $\widetilde{W}_{\mathrm{O}}=0.1$, the dashed curve corresponds to $\widetilde{W}_{\mathrm{O}}=0.2$ and the solid one corresponds to $\widetilde{W}_{\mathrm{O}}=0.3$.

and

$$
\begin{aligned}
\gamma(k) & =\left[\frac{3}{64 \pi} \frac{n_{\mathrm{e}}}{\lambda v_{\mathrm{t}}^{2}} \frac{c^{2}}{\omega_{\mathrm{p}}^{2}} k^{2}\right. \\
& \left.+p \frac{3 \sqrt{2^{5}}}{5} \lambda v_{\mathrm{t}} k^{2} \int_{0}^{\infty} y^{9 / 4} G(y, k \lambda) f_{2} \mathrm{~d} y\right] / D,
\end{aligned}
$$

where $D=\int_{0}^{\infty} y^{3} F(y, k \lambda) \frac{\partial f_{0}}{\partial y} \mathrm{~d} y$ and $v_{\mathrm{t}}=\sqrt{T / m_{\mathrm{e}}}$ is the electron thermal velocity.

The functions $F$ and $G$ are calculated with a precision better than $5 \%$ using numerical fits of the continued fractions [16], so:

$$
F=\left[\left(1+\left(\frac{\alpha}{\delta}\right)^{2}\right)^{-1 / 2}\right] / 2
$$

and

$$
G=2\left(1+\alpha^{2} \theta\right) /\left[3\left(1+\alpha^{2} \beta\right)(1+2 F)\right],
$$

where $\alpha=8 k \lambda y^{2}, \delta=3 \pi / 2, \theta=30 \beta / \delta^{2}$ and $\beta=$ $\left(5 \delta^{2} 252-3 / 4\right) /\left(\delta^{2}-30\right)$.

$p=-1$ for the linearly laser polarized laser pulse and $p=+2$ for the circularly laser polarized pulse. The first term in the right hand side of the Eq. (7) corresponds to the attenuation of the wave and the second term corresponds to the Weibel source.

The growth rate of the most unstable Weibel mode and its group velocity are computed from this relation as:

$$
\begin{aligned}
& \gamma_{\max }=\frac{2^{15 / 4}}{3^{3 / 2} \sqrt{\pi}} \frac{v_{\mathrm{t}}^{5 / 2}}{\sqrt{n_{\mathrm{e}}}} \frac{\omega_{\mathrm{p}}}{c} \frac{\left[\int_{0}^{\infty} \sqrt{y} f_{2} \mathrm{~d} y\right]^{3 / 2}}{\int_{0}^{\infty} f_{0} \mathrm{~d} y}, \\
& v_{\mathrm{g}}=\frac{v_{\mathrm{t}}}{\sqrt{2}} \frac{\int_{0}^{\infty} \sqrt{y} f_{1} \mathrm{~d} y}{\int_{0}^{\infty} f_{0} \mathrm{~d} y} .
\end{aligned}
$$

By considering the explicit expressions of $f_{1}$ (Eq. (4)) and $f_{2}$ (Eq. (5)), the above equations can be written as functions

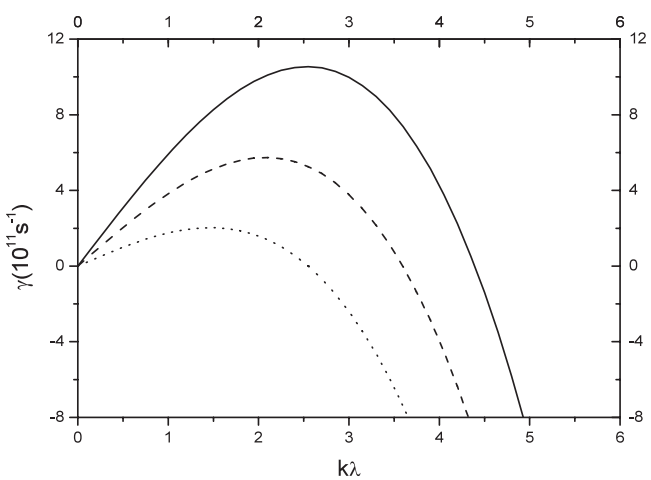

Fig. 2 Weibel instability growth rate spectrum: $\gamma(k \lambda) . k$ is the wave number of the Weibel mode and $\lambda$ is the mean free path of the electron. The dotted curve corresponds to $\widetilde{W}_{\mathrm{O}}=0.01$, the dashed one corresponds to $\widetilde{W}_{\mathrm{O}}=0.02$ and the solid one corresponds to $\widetilde{W}_{\mathrm{O}}=0.03 . T=1 \mathrm{keV}$ and $n_{\mathrm{e}}=10^{21} \mathrm{~cm}^{-3}$

of the scaling parameter, $\widetilde{W}_{\mathrm{O}}$, so:

$$
\begin{aligned}
\frac{\gamma_{\max }}{10^{11} \mathrm{~s}^{-1}}= & 4.416 \times 10^{3} \sqrt{\frac{T_{\perp}}{\mathrm{keV}}} \sqrt{\frac{n_{\mathrm{e}}}{\mathrm{cm}^{-3}}} \\
& \times \frac{\widetilde{W}_{0}^{3 / 2}}{\left(1+\widetilde{W}_{\mathrm{O}}\right)^{-1 / 4}+\frac{1}{3} \widetilde{W}_{\mathrm{O}}\left(1+\widetilde{W}_{\mathrm{O}}\right)^{-3 / 4}}, \\
v_{\mathrm{g}}=0 . &
\end{aligned}
$$

Numerical analysis of this set of Eqs. (1)-(11) permits to analyze the Weibel instability due to the laser pulse field in the laser fusion plasma corona.

Because $v_{\mathrm{g}} \sim f_{1}=0$, the excited Weibel modes by this mechanism are not convective. However, other Weibel sources, such as those due to temperature and density gradients in the corona, can contribute to the convection of these modes. We present, on Fig. 2, the Weibel instability growth rate spectrum $\gamma(k \lambda)$, where $k$ is the Weibel mode 


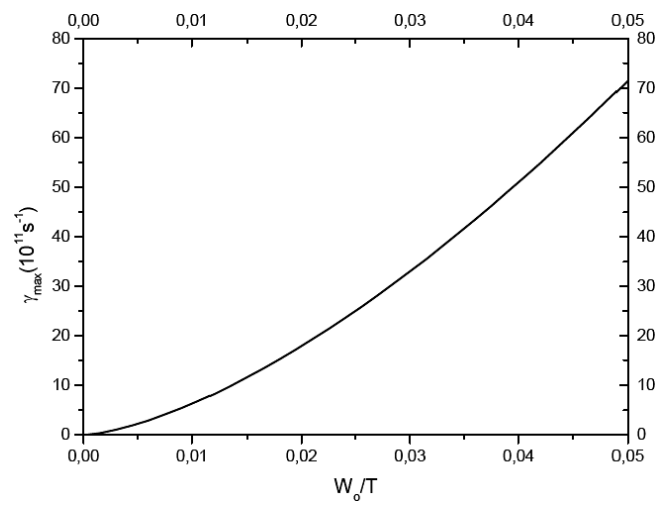

Fig. $3 \gamma_{\max }$ as function of the scaling parameter, $\widetilde{W}_{\mathrm{O}}=\frac{W_{\mathrm{O}}}{T} \cdot T_{\mathrm{e}}=$ $1 \mathrm{keV}$ and $n_{\mathrm{e}}=10^{21} \mathrm{~cm}^{-3}$

wave number and $\lambda$ is the electron mean free path. We also present, in Fig. 3, the most unstable Weibel mode, $\gamma_{\max }$, as a function of the scaling parameter, $\widetilde{W}_{\mathrm{O}}$. These figures show that the unstable Weibel modes are non collisionals, $k \lambda>1$. The growth rate of the most unstable Weibel mode (Eq. (10)) is $\gamma_{\max } \geq 10^{11} \mathrm{~s}^{-1}$ in the vicinity of the critical layer, $\omega_{\mathrm{p}}=\omega_{\mathrm{L}}$. We point out that the growth rate calculated in this model yields back results of the Fokker-Planck simulations especially for low values of the scaling factor: $\widetilde{W}_{\mathrm{O}} \ll 1$.

\section{Scaling Laws}

In the inertial target fusion experiments, the created plasma parameters are linked to the parameters of the incident laser pulse to the target. In Ref. [17], a scaling law of the electron temperature is established. This law is obtained by computation of the energy stock in the critical layer, so:

$$
\frac{T_{\mathrm{ec}}}{\mathrm{keV}}=4.3\left(\frac{I_{\mathrm{a}}}{10^{14} \mathrm{~W} / \mathrm{cm}^{2}}\right)^{2 / 3}\left(\frac{\lambda_{\mathrm{L}}}{\mu \mathrm{m}}\right)^{4 / 3},
$$

where $T_{\mathrm{ec}}, I_{\mathrm{a}}$ and $\lambda_{\mathrm{L}}$ are the electron temperature in the critical layer, the absorbed laser intensity and the laser wave length, respectively.

This Eq. (12) corresponds to the thermal energy indirectly deposited by different absorption mechanisms which are the inverse bremsstrahlung absorption, the resonance absorption and the anomalous absorption. We point out that the heating via these absorption mechanisms is isotropic.

However, the electron quiver energy in the laser electric field (Eq. (2)) corresponds to the direct effect of the laser pulse on the plasma electrons (direct heating). It is an anisotropic heating in the direction of the laser electric field.

In the laser inertial fusion experiments, the indirect heating is important compared to the direct heating due to the electron quiver energy in the laser electric field. This is justified by the fact that the electron temperature, $T \sim$
$\mathrm{keV}$, is largely greater than the photon energy, $\hbar \omega_{\mathrm{L}} \sim \mathrm{eV}$ : $T \gg \hbar \omega_{\mathrm{L}}$.

The electron density in the critical layer, $n_{\mathrm{c}}$, is given by:

$$
\frac{n_{\mathrm{c}}}{\mathrm{cm}^{-3}}=1.1 \times 0^{21}\left(\frac{\lambda_{\mathrm{L}}}{\mu \mathrm{m}}\right)^{-2} .
$$

The collision frequency in the critical layer is given by the temperature, $T_{\mathrm{e}}$, the density, $n_{\mathrm{e}}$, and the plasma ionization number, $Z$, as:

$$
\frac{\vartheta_{\mathrm{c}}}{\mathrm{s}^{-1}}=3.4 \times 10^{-9}(Z+1) \frac{n_{\mathrm{e}}}{\mathrm{cm}^{-3}}\left(\frac{T_{\mathrm{e}}}{\mathrm{keV}}\right)^{-3 / 2} \ln \Lambda .
$$

In $\Lambda$ means the Coulomb logarithm. In the case of the laser fusion plasma, $\ln \lambda \approx 10$.

By taking into account the Eqs. (13), (14), the collision frequency in the critical layer for an isothermal corona can be expressed as a function of the laser parameters as:

$$
\begin{aligned}
& \vartheta_{\mathrm{c}}\left(\mathrm{s}^{-1}\right) \approx 4.2 \times 10^{11}(Z+1)\left(\frac{I_{\mathrm{a}}}{10^{14} \mathrm{~W} / \mathrm{cm}^{2}}\right)^{-1}\left(\frac{\lambda}{\mu \mathrm{m}}\right)^{-4}, \\
& \frac{\vartheta_{\mathrm{c}}}{\omega_{\mathrm{L}}} \approx 2.2 \times 10^{-5}(Z+1)\left(\frac{I_{\mathrm{a}}}{10^{14} \mathrm{~W} / \mathrm{cm}^{2}}\right)^{-1}\left(\frac{\lambda}{\mu \mathrm{m}}\right)^{-3} .
\end{aligned}
$$

This equation shows that the collisions are efficient at shorter laser wavelength.

The scaling parameter (Eq. (2)) is given by:

$$
\begin{aligned}
\widetilde{W}_{\mathrm{O}}= & 2.16 \times 10^{-3}\left(\frac{I}{10^{14} \mathrm{~W} / \mathrm{cm}^{2}}\right) \\
& \times\left(\frac{I_{\mathrm{a}}}{10^{14} \mathrm{~W} / \mathrm{cm}^{2}}\right)^{-2 / 3}\left(\frac{\lambda}{\mu \mathrm{m}}\right)^{2 / 3} \\
& \times\left[1-5 \times 10^{10}(Z+1)^{2}\left(\frac{I_{\mathrm{a}}}{10^{14} \mathrm{~W} / \mathrm{cm}^{2}}\right)^{-2}\left(\frac{\lambda}{\mu \mathrm{m}}\right)^{-6}\right],
\end{aligned}
$$

$I_{\mathrm{a}}=A I_{\mathrm{L}}$, where $I_{\mathrm{L}}$ is the laser pulse intensity and $A$ is the absorption coefficient.

The absorption coefficient $[8,9]$ due to the inverse bremsstrahlung mechanism, for a linear density spatial profile, is obtained in the WKB approximation by:

$$
A_{\mathrm{IB}}=1-\exp \left(-\frac{32}{15} \frac{v_{\mathrm{t}}}{c} \frac{L_{\mathrm{n}}}{\lambda}\right) .
$$

$L_{\mathrm{n}}=\left|\frac{\vec{\nabla} n_{\mathrm{e}}}{n_{\mathrm{e}}}\right|^{-1}$ is the density gradient length.

In the case where we can consider a monodimensional corona expansion, the density profile is characterized by a density gradient length proportional to the expansion velocity and to the laser pulse duration, $\tau$, so:

$L_{\mathrm{n}} \approx C_{\mathrm{S}} \tau$, where $C_{\mathrm{S}}=\sqrt{\frac{Z T_{\mathrm{e}}}{m_{\mathrm{i}}}}$ is the sound speed in the coronal plasma. $m_{\mathrm{i}}$ means the ion mass. 

law:

Using Eq. (12), $L_{\mathrm{n}}$ can be given by the following scale

$$
\frac{L_{\mathrm{n}}}{\mu \mathrm{m}} \approx 9.4 \times 10^{11}\left(\frac{I_{\mathrm{a}}}{10^{14} \mathrm{~W} / \mathrm{cm}^{2}}\right)^{1 / 3}\left(\frac{\lambda}{\mu \mathrm{m}}\right)^{2 / 3} \frac{\tau}{s} .
$$

The inverse bremsstrahlung absorption is then expressed as:

$$
\begin{aligned}
A_{\mathrm{IB}} \approx 1-\exp [ & -5.51 \times 10^{2}\left(\frac{I_{\mathrm{a}}}{10^{14} \mathrm{~W} / \mathrm{cm}^{2}}\right)^{2 / 3} \\
& \left.\times\left(\frac{\lambda}{\mu \mathrm{m}}\right)^{4 / 3} \frac{\tau}{\mathrm{ns}}\right] .
\end{aligned}
$$

Other mechanisms participate to the absorption of the laser energy in the corona; namely the resonance absorption mechanism in the vicinity of the critical layer. Subsequently, the effective absorption be greater than that due to the inverse bremsstrahlung mechanism (Eq. (20)), $A>A_{\mathrm{IB}}$. Then, in the laser fusion experiments using nanosecond laser pulses with laser wavelength $\lambda_{\mathrm{L}} \leq$ micrometer, we can consider in a good approximation that the laser pulse energy is totally absorbed with $I_{\mathrm{a}}=I_{\mathrm{L}}$ and $A=1$.

It is important to express the local laser intensity, as a function of the laser pulse parameters. The spatial evolution, in the corona, of the laser electric field magnitude, $E_{0}(x)$, in the case of a linear density profile is given by the Ai Airy function $[8,9,14]$, hence:

$$
E_{0}(x)=2 \sqrt{\pi}\left(\frac{\omega_{\mathrm{L}} L_{\mathrm{n}}}{c}\right)^{1 / 6} E_{\mathrm{v}} A \mathrm{i}(\xi) \exp \left(-\frac{\vartheta_{\mathrm{c}}(x)}{\omega_{\mathrm{L}}}\right),
$$

where $E_{\mathrm{v}}$ is the laser electric field magnitude in the interface vacuum-plasma $\left(x=L_{\mathrm{n}}\right)$, and $\xi=$ $\left(\frac{\omega_{\mathrm{L}} L_{\mathrm{n}}}{c}\right)^{2 / 6}\left(\frac{x}{L_{\mathrm{n}}}+\mathrm{i} \frac{\vartheta_{\mathrm{c}}(x)}{\omega_{\mathrm{L}}}\right)$ is a dimensionless spatial coordinate. by:

The laser intensity in the critical layer, $x=0$, is given

$$
I_{\mathrm{c}}=4 \pi\left(\frac{\omega_{\mathrm{L}} L_{\mathrm{n}}}{c}\right)^{2 / 6} I_{\mathrm{v}} A \mathrm{i}^{2}(0) \exp \left(-\frac{2 \vartheta_{\mathrm{c}}(0)}{\omega_{\mathrm{L}}}\right) .
$$

By taking into account the Eqs. (15), (16), (19), the laser intensity in the critical layer can be expressed as:

$$
\begin{aligned}
\frac{I_{\mathrm{c}}}{\frac{10^{14} \mathrm{~W}}{\mathrm{~cm}^{2}}}= & 2.8 \times 10^{4} \frac{I_{\mathrm{v}}}{\frac{10^{14} \mathrm{~W}}{\mathrm{~cm}^{2}}}\left(\frac{I_{\mathrm{a}}}{\frac{10^{14} \mathrm{~W}}{\mathrm{~cm}^{2}}}\right)^{1 / 9}\left(\frac{\lambda_{\mathrm{L}}}{\mu \mathrm{m}}\right)^{-1 / 9}\left(\frac{\tau}{\mathrm{s}}\right)^{1 / 3} \\
& \times \exp \left[-4.4 \times 10^{-5}(Z+1)\left(\frac{I_{\mathrm{a}}}{\frac{10^{14} \mathrm{~W}}{\mathrm{~cm}^{2}}}\right)^{-1}\left(\frac{\lambda_{\mathrm{L}}}{\mu \mathrm{m}}\right)^{-3}\right] .
\end{aligned}
$$

The Eqs. (10), (12), (13), (17) allow us to establish a scaling law for the growth rate of the most unstable Weibel mode in the critical layer as:

$$
\begin{aligned}
\gamma_{\max }= & 1.4 \times(2-A-2 \sqrt{1-A})^{3 / 2} A^{-1 / 2} I_{\mathrm{L}} \lambda_{\mathrm{L}}^{1 / 2} \tau^{1 / 2} \\
& \times \exp \left[-6.6 \times 10^{-5}(Z+1) A^{-1} I_{\mathrm{L}}^{-1} \lambda_{\mathrm{L}}^{-3}\right] .(24)
\end{aligned}
$$

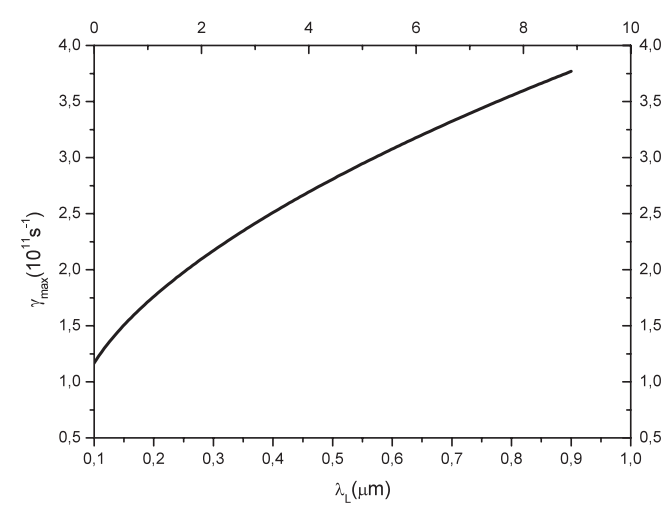

Fig. $4 \gamma_{\max }$ as function of the laser wavelength, $\lambda_{\mathrm{L}} \cdot \tau=1 \mu \mathrm{m}$, $Z=10$ and $I_{\mathrm{L}}=10^{15} \mathrm{~W} / \mathrm{cm}^{3}$

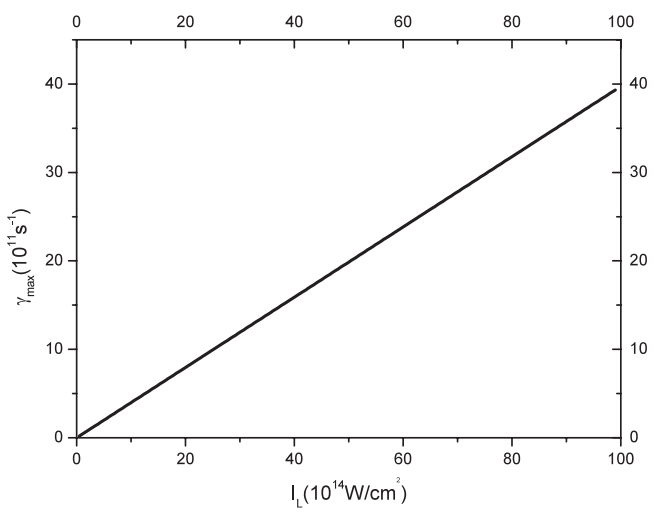

Fig. $5 \gamma_{\max }$ as function of the laser intensity, $I_{\mathrm{L}} \cdot \tau=1 \mathrm{~ns}, Z=10$ and $\lambda_{\mathrm{L}}=1 \mu \mathrm{m}$

Here $I_{\mathrm{L}}$ is the laser pulse intensity and $A$ is the absorption coefficient. With the intention of $I_{\mathrm{v}}=(2-A-2 \sqrt{1-A}) I_{\mathrm{L}}$. Note that $I_{\mathrm{L}}$ in $10^{14} \mathrm{~W} / \mathrm{cm}^{2}, \lambda_{\mathrm{L}}$ in $\mu \mathrm{m}$ and $\gamma_{\max }$ in $10^{11} \mathrm{~s}^{-1}$.

We have presented on the Fig. 4 the $\gamma_{\max }$ as a function of the laser pulse intensity, $I_{\mathrm{L}}$, and, on the Fig. 5, we have presented the $\gamma_{\max }$ as function of the laser wavelength, $\lambda_{\mathrm{L}}$.

In the case of the total absorption, $A=1$, the above expression is simplified as:

$$
\gamma_{\max }=1.4 I_{\mathrm{L}} \lambda_{\mathrm{L}}^{1 / 2} \tau^{1 / 2} \exp \left[-6.6 \times 10^{-5}(Z+1) I_{\mathrm{L}}^{-1} \lambda_{\mathrm{L}}^{-3}\right] .
$$

We point out from this that $\gamma_{\max } \sim I_{\mathrm{L}} \lambda_{\mathrm{L}}^{1 / 2} \tau^{1 / 2}$. This corresponds to the results of the Ref. [6] using the FokkerPlanck theory. It is in good agreement with the laser fusion experiments which show a relatively stability for the short laser pulse such as in fast ignition experiments using the femto laser pulses. The resulting scaling laws permit to optimize the laser parameters using in the fusion experiments in order to minimize the energy losses due to the Weibel instability. 


\section{Conclusion}

In the present work, the Weibel instability is studied in the corona of the laser fusion plasma through a theoretical model. The unperturbed electronic distribution function is supposed to be a local bi-Maxwellian. The theoretical model presented in this paper show highly unstable Weibel modes in the vicinity of the critical layer: $\gamma_{\max }>10^{11} \mathrm{~s}^{-1}$.

Practical scaling laws are established for the instability parameters. The growth rate of the most unstable Weibel mode, $\gamma_{\max }$, is proportional to $I_{\mathrm{L}} \lambda_{\mathrm{L}}^{1 / 2} \tau^{1 / 2}$. The results of this paper are in good agreement with the results of the Ref. [6] founded in the frame of the kinetic FokkerPlanck theory. Also the dependence of $\gamma_{\max }$ on $\tau^{1 / 2}$ is in agreement with the laser fusion experiments. The instability is then reduced when the used laser pulse is shorter like in fast ignition schemes.

The saturation of the Weibel instability by the nonlinear effects and generated magnetic field is not studied in this paper.

We expect that the theoretical study presented on this paper permits to optimize the laser pulse parameters in order to have minimum energy losses in the laser fusion experiments.
[1] E.S. Weibel, Phys. Rev. Lett. 2, 83 (1959).

[2] O. Nobuhiro and H. Makoto, J. Korean Astro. Soc. 37, 547 (2004).

[3] B. Shokri and M. Ghorbanalili, Phys. Plasmas 11, 5398 (2004).

[4] J.M. Wallace and E.M. Epperlein, Phys. Fluids B 3, 1579 (1991).

[5] A. Ramani and J. Laval, Phys. Fluids 28, 980 (1978).

[6] K. Bendib, A. Bendib and A. Sid, Laser Part. Beams 16, 423 (1998).

[7] A. Bendib, K. Bendib and A. Sid, Phys. Rev. E 55, 7522 (1997).

[8] H. Hora, Laser Plasma and Nuclear Energy (New York Plenum Press. 1975).

[9] L. Ginsburg, Propagation of Electromagnetic Waves in Plasmas (Cordon and Breach, N. Y., 1960).

[10] A. Sid, Phys. Plasmas 10, 214 (2003).

[11] S.I. Braginski, in Reviews of Plasmas Physics (M.A. Leonvitch, Consultant Bureau, N. Y. 1985, Vol. 1).

[12] V.P. Silin, Sov. Phys. JETP 20, 1510 (1965).

[13] A. Bruce Langdon, Phys. Rev. Lett. 44, 575 (1980).

[14] M. Abramowitz and I.A. Stegun, Handbook of Mathematical Functions (Dover, New York, 1965).

[15] J.M. Wallace, et al., Phys. Fluids 30, 4, 1085 (1987).

[16] J.P. Matte, A. Bendib and J.F. Luciani, Phys. Rev. Lett. 58, 2067 (1987).

[17] R. Fabro, C.F. Max and E. Fabre, Phys. Fluids 28, 1463 (1985). 